\title{
CFRP 复合材料铣削力、温度及表层损伤分析
}

\author{
王福吉 ${ }^{1}$ 殷俊伟 ${ }^{1} \quad$ 贾振元 $^{1}$ 马建伟 ${ }^{1}$ 徐震宇 $^{2}$ 王 东 $^{1}$ \\ (1. 大连理工大学精密与特种加工教育部重点实验室 大连 116024; \\ 2. 中国兵器工业集团内蒙古第一机械集团有限公司 包头 014030)
}

\begin{abstract}
摘要: 碳纤维增强树脂基复合材料(Carbon fiber reinforced polymer, CFRP)以其轻质、高强等优点, 在航空航天高端装备上展 现出巨大优势。然而, CFRP 中各组成相所需的切削能量及热导率具有差异性, 在切断高强纤维的同时极易造成低强的纤维/ 树脂界面发生开裂, 而热量积聚所引起的高温又会使树脂软化加剧裂纹扩展, 严重影响装备的服役性能和可靠性。阐明切削 力和切削热对加工损伤的影响机制是实现 CFRP 高质高效加工的关键。基于数字图像处理技术，建立 CFRP 铁削加工表层损 伤面积评价方法, 该方法可有效避免传统方法因只能考虑损伤的一维长度信息而无法评价加工损伤程度的问题。分析主轴转 速和每齿进给对表层损伤面积、切削力以及切削温度的影响规律, 并讨论切削力和切削温度对表层损伤面积因子的影响。研 究表明: 通过减小单次切削厚度和控制切削温度在适合区间可有效降低加工损伤。
\end{abstract}

关键词: 碳纤维复合材料; 铣削; 损伤; 切削力; 切削温度

中图分类号: TB332

\section{Measurement and Analysis of Cutting Force, Temperature and Cutting-induced Top-layer Damage in Edge Trimming of CFRPs}

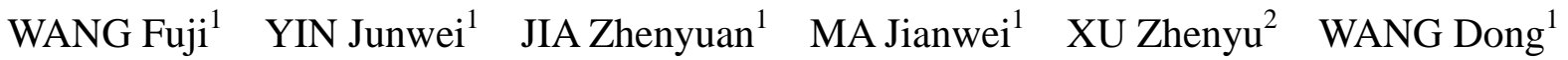

(1. Key Laboratory for Precision and Non-traditional Machining Technology of the Ministry of Education, Dalian University of Technology, Dalian 116024;

2. Inner Mongolia First Machinery Group Corporation Limited, Baotou 014030)

\begin{abstract}
Carbon fiber reinforced polymer (CFRP) composites have a great advantage in the fields including aeronautics and astronautics for their light weight and high strength, when compared to the more conventional materials. However, the cutting energy and the heat conductivity are serious difference between fibers and resin phases. Consequently, when cutting off the high-strength fibers, it is prone to provide problems like crack for the low-strength interface. Besides, heat accumulation may cause resin softening, which in turn deteriorates the crack growth. In some cases, the crack has a strong impact on the service performance and reliability of final parts. To solve the problems encountered in realizing the high quality and efficiency when machining of CFRP composites, the mechanism of damage formation under the combined effects of cutting force and cutting heat should be clarified first. In the current paper, a novel method to evaluate the damage extent on the top layer of CFRP composites is developed. This method can replace the conventional assessment approach of damage extent, which can only taking the maximum length of damage into account. Afterwards, the influences of cutting parameters on the damage, cutting force and cutting temperature are analyzed. The effects of cutting force and cutting temperature on the damage formation is also discussed. It suggests that reducing the single cutting thickness and controlling the cutting temperature in the reasonable range are the effective approach to improve machining quality.
\end{abstract}

Key words: carbon fiber reinforced polymer (CFRP); edge trimming; damage; cutting force; cutting temperature

\section{0 前言}

碳纤维增强环氧树脂基复合材料(Carbon fiber

* 国家自然科学基金(U1508207, 51575082, 51621064)、国家 973 计划课题 (2014CB046503)、基本科研业务费(DUT16TD01)和国家科技重大专项 (2016ZX04001-002) 资助项目。20170630 收到初稿, 20170920 收到修 改稿 reinforced polymer, CFRP)以其轻质、高强、可整体 制造等突出优点, 在航空航天领域高端装备上展现 出巨大优势, 其用量也已成为衡量飞机先进性的重 要标志 ${ }^{[1-3]}$ 。然而, CFRP 是公认的难加工材料, 宏 观呈非均质、各向异性、层叠特征, 细观呈纤维、 树脂、界面构成的多相混合态, 其切削过程的实质 是切削力和切削热在层间以及相间传递的复杂过 
程。此外, 各相所需的切削能量及热导率具有差异 性, 在切断高强纤维的同时极易造成低强的纤维/ 树脂界面发生开裂, 而热量积聚所引起的高温又会 使树脂软化加剧裂纹扩展, 严重影响飞机的服役性 能和可靠性。据统计, 在飞机的装配环节, 因加工 损伤超差导致的 CFRP 复合材料构件不合格率约占 $60 \%{ }^{[4]}$ 。因此, 必须从根源上阐明切削力和切削热 共同作用下加工损伤现象背后的机理, 为实现 CFRP 复合材料高质高效加工提供理论依据。

近年来, 国内外学者竞相对 CFRP 复合材料加 工过程进行研究, 并取得了一定成果。其中, 在理 论研究方面, 最具代表性的是 HOCHENG 等 ${ }^{[5-6]}$ 基 于线弹性断裂力学建立的钻削分层损伤临界轴向力 解析模型, 该模型的建立证明了钻削轴向力过大是 导致出口分层损伤的主要原因, 从而为钻削出口分 层损伤抑制方法的确定奠定了坚实的理论基础。利 用该模型, 他们分析了麻花钻、锯钻、烛台钻和套 料钻等不同刀具结构形式对分层临界轴向力的影 响。JAIN 等 ${ }^{[7-8]}$ 对上述模型进行了改进, 突破性的 将材料各向异性对临界轴向力的影响考虑在内, 使 模型更符合真实情况。此外, 针对铣削 CFRP 复合 材料时, 表层材料损伤严重的问题, HINTZE 等 ${ }^{[9]}$ 基于Lekhnitskii 的各向异性弹性理论以及 Puck 失效 准则, 计算分析了刀齿旋转瞬间的面下损伤深度。 陈燕等 ${ }^{[10]}$ 基于 Hintze 模型, 进一步分析了每齿切削 长度对毛刺产生的影响。上述理论模型都是从力学 角度, 对加工过程进行了准静态分析, 结果表明, 切削力是影响 CFRP 复合材料加工损伤产生的主要 因素之一。

在仿真研究方面, SOLDANI 等 ${ }^{[11-12]}$ 采用宏观 等效均质建模方法同时考虑材料的各向异性, 分析 了面下损伤深度、切削力随着纤维方向、刀具几何 以及切深的变化规律。RAO 等 ${ }^{[13-14]}$ 采用细观多相建 模方法, 建立了包含纤维、树脂及界面的细观模型, 利用该仿真模型, 分析发现切削过程中纤维失效以 压溃和弯曲断裂为主。殷俊伟等 ${ }^{[15]}$ 首次建立了可以 模拟复合材料连续切削过程的二维宏观仿真模型,

分析了直角切削不同纤维方向 CFRP 单向板时的面 下损伤深度变化规律。然而, 上述仿真模型均未考 虑切削热对加工过程的影响, 造成模型的普适性差。

通过试验, YASHIRO 等 ${ }^{[16]}$ 系统地分析了 CFRP 复合材料铣削温度随铣削参数的变化规律, 结果表 明当铣削速度超过 $300 \mathrm{~m} / \mathrm{min}$ 以后, 最高铣削温度 出现了饱和现象, 即随着铣削速度的继续增加, 最高铣削温度的增速显著放缓。BRINKSMEIER 等 ${ }^{[17-18]}$ 发现纤维的弯曲变形程度与加工过程中的热
影响区深度有关。PECAT 等 ${ }^{[19]}$ 认为当工件温度接近 树脂的玻璃转化温度时, 可显著降低加工引起的面 下裂纹扩展。上述文献研究表明, 切削热是 CFRP 复合材料加工损伤产生的主要影响因素之一。综上 所述, 阐明切削力和切削热对加工损伤的影响机制 是实现 CFRP 复合材料高质高效加工的关键。

然而, 经检索国内外未见有关切削力、热共同 作用下 CFRP 复合材料加工损伤产生机制方面的文 献报道。但是, 实际切削过程中, 切削力和切削热 始终是同时存在的, 二者既相互影响又相互制约。

因此, 本文以侧铣 CFRP 多向板为例, 首先提出了 一种基于数字图像处理技术的 CFRP 铣削表层损伤 面积快速提取方法, 弥补了人工测量依赖性大的不 足。同时结合测量得到的铣削力和铣削温度, 系统 分析了主轴转速和每齿进给对铣削力、铣削温度以 及损伤因子的影响规律, 提出了 CFRP 复合材料加 工表层损伤的抑制方法, 为 CFRP 高质高效加工工 具及工艺的开发奠定了基础。

\section{1 表层加工损伤面积评价}

\section{1 损伤因子计算模型}

CFRP 复合材料侧铣加工过程中，损伤区域多 位于材料表层, 形状不规则、分布无规律, 且损伤 类型具有非单一性。如何对 CFRP 加工损伤程度进 行准确表征是加工过程分析中所面临的一大挑战性 难题。目前, 最大损伤长度是使用最为广泛的一种 加工损伤程度评价方法 ${ }^{[20-22]}$ 。该方法虽然具有直观 易用、操作简单的优点, 但是, 其只包含了加工损 伤的一维信息, 无法全面、准确、真实地表征 CFRP 复合材料的加工损伤程度, 极易出现加工损伤程度 明显不同的两种情况却具有相同的损伤长度, 如图 1 所示, 这显然是不合理的。

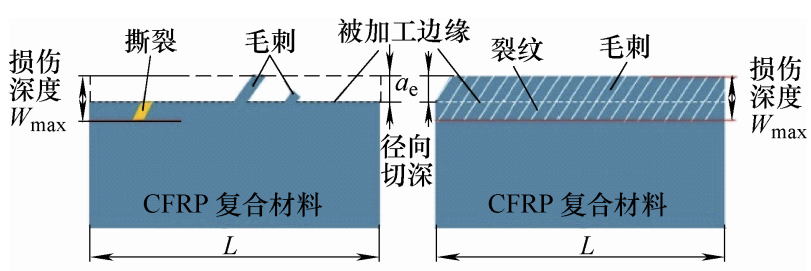

(a) 损伤零星分布

(b) 损伤均匀分布

图 1 一维损伤评价方法示意图

鉴于此，本文提出了基于损伤区域面积大小的 二维加工损伤评价新方法, 既包含了最大损伤长度、 损伤的几何形状及其分布信息，同时还弥补了传统 一维损伤评价方法区分度低的不足。在此基础上, 建立了面积损伤因子计算模型，其表达式为 


$$
F_{\mathrm{D}}=\frac{A_{\mathrm{D}}}{A_{\mathrm{Q}}}
$$

式中, $F_{\mathrm{D}}$ 代表面积损伤因子, 该值的大小可直接反 映加工损伤程度的大小; $A_{\mathrm{D}}$ 表示损伤区域的面积; $A_{\mathrm{Q}}$ 为侧铣加工时表层被去除材料的面积, 且 $A_{\mathrm{Q}}=a_{\mathrm{e}} \times L$, 其中, $a_{\mathrm{e}}$ 为侧铣加工时的径向切深, $L$ 是工件被加工边缘的长度。

\section{2 损伤面积提取方法}

为了表征侧铣 CFRP 复合材料时表层损伤区域 的面积大小，提出了基于数字图像处理技术的损伤 区域图像提取方法, 弥补了人工测量依赖性大的不 足, 具体操作流程如图 2 所示。加工前和加工后的 工件表层图像通过彩色扫描仪(Microtek ScanMaker 3860)获得, 扫描分辨率采用 $1200 \mathrm{dpi}$ 。在开始图像 扫描之前, 需要对扫描仪进行标定, 以获得图像中 每个像素所占的面积大小。本文借鉴了视觉测量中 常用的高精度国际棋盘格标定方法, 标定结果为 4.6 $\times 10^{-4} \mathrm{~mm}^{2} /$ 像素。

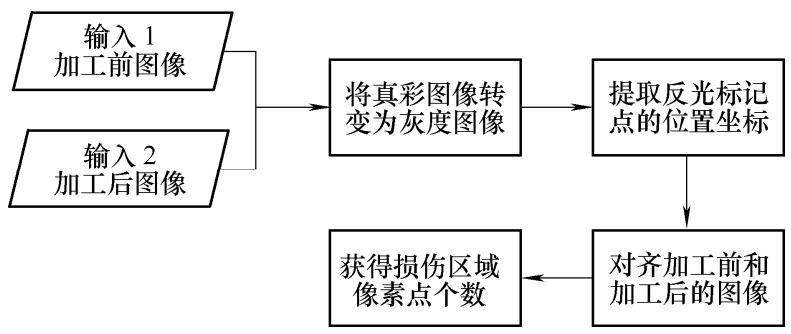

图 2 损伤区域图像的提取流程

由于数字图像可以被定义为一个二维矩阵, 其 中第 $i$ 行第 $j$ 列位置上的元素通常被定义为图像在这 一位置的亮度。若相同位置处, 加工前后两次扫描 结果具有不同的亮度值, 则说明在该位置处产生了 损伤。相反, 若相同位置处前后两次扫描的结果一 致, 则说明该位置处并未出现损伤。因此, 为了方 便提取图像中的损伤信息, 可以先将加工前和加工 后的两幅图像进行减法运算。为了确保加工前和加 工后图像在对准时的精确度, 需要在工件表面上粘 贴反光标记点。然后, 根据 Otsu 的自动阈值选择法 对图像相减后的结果进行二值化处理并取反, 效果 如图 3 所示。上述图像处理过程是在 Matlab 软件平 台上通过编程加以实现。

最后, 统计所有亮度值大于零的像素点个数, 即为加工损伤区域所包含的像素点总数。将统计得 到的损伤区域像素点总数乘以扫描仪每个像素所占 的面积, 便可得到加工损伤区域的面积。具体的计 算表达式为

$$
A_{\mathrm{D}}=N(\text { 像素 }) \times 4.6 \times 10^{-4} \mathrm{~mm}^{2} / \text { 像素 }
$$

式中, $N$ 代表损伤区域所包含的像素点总数。

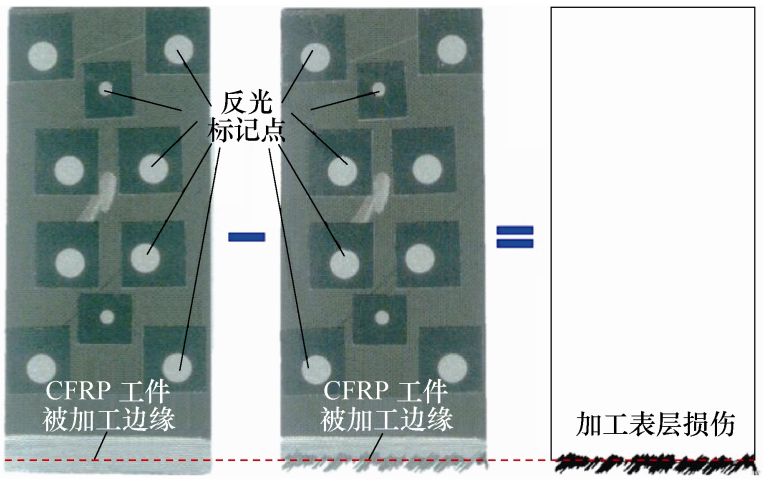
(a) 加工前
(b) 加工后
(c) 损伤区

图 3 损伤区域图像的提取效果示例

\section{2 试验材料及装置}

为了研究 CFRP 复合材料的加工过程, 在高速 加工中心(Mikron HSM 500)上开展了一系列侧铣试 验, 装置如图 4 所示。试验中所使用的工件材料为 T800/P2352 碳纤维增强环氧树脂基复合材料多向层

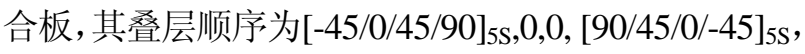
纤维含量为 $60 \%$, 工件尺寸为 $100 \mathrm{~mm} \times 50 \mathrm{~mm} \times$ $5.25 \mathrm{~mm}$ 。铣削时刀具沿着工件的 $50 \mathrm{~mm}$ 长边缘侧 不断向前进给, 且刀具的瞬时切削速度方向与刀具 进给方向相同, 而刀具的进给方向又与表层的纤维 方向呈 $135^{\circ}$ 夹角。

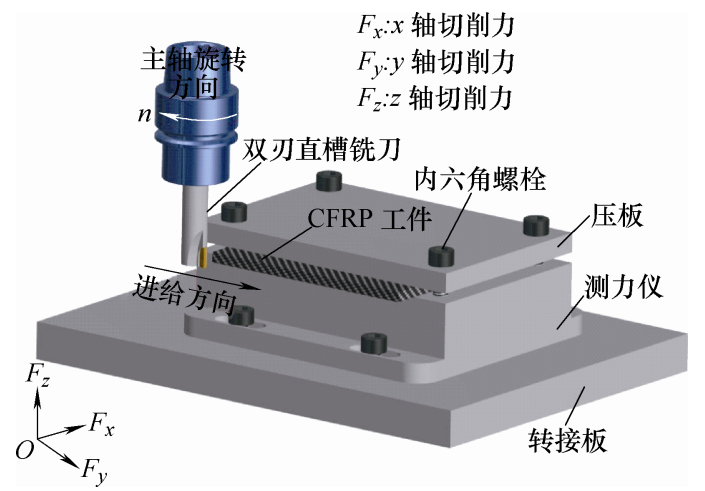

图 4 试验装置示意图

试验中所使用的刀具是双刃直槽聚晶金刚石 (Polycrystalline diamond, PCD)铣刀, 直径 $10 \mathrm{~mm}$, 具有 $0^{\circ}$ 螺旋角、 $4^{\circ}$ 前角和 $9^{\circ}$ 后角。所有试验均在干 式切削条件下完成, 详细的切削参数见表 1 , 其他 数均为常数, 径向切深取 $2.5 \mathrm{~mm}$ 。此外, 铣削力分 量随时间的演化规律采用压电式三分量测力计(瑞 士 Kistler 9257B)进行实时在线测量, 采样频率设为 $20 \mathrm{KHz}$ 。铣削温度随时间的变化规律通过高速红外 热成像仪(加拿大 Telops TS-IR)实时记录, 焦距固定 为 $1 \mathrm{~m}$ 。为排除偶然性因素对试验结果的影响, 每 组试验重复 1 次。 
表 1 侧铣加工参数

\begin{tabular}{cc}
\hline 主轴转速(r/min) & 每齿进给 $(\mathrm{mm} / \mathrm{tooth})$ \\
\hline 2500 & 0.01 \\
5000 & 0.05 \\
10000 & 0.10 \\
\hline
\end{tabular}

\section{3 结果与讨论}

\section{1 损伤面积变化规律}

在每次试验开始前, 需要先扫描一次 CFRP 复合材料工件的表面, 以记录加工前工件表面的 图像信息; 每次铣削加工后, 需要再次扫描该表 面, 记录加工后的图像信息。接下来, 根据本文 第 1 部分所述的加工损伤区域图像提取方法, 分 别对每组试验中加工前和加工后的图像进行处 理, 即可得到不同铣削参数下的加工损伤区域所
包含的像素点个数, 按照式(2)经过简单计算便可 得到损伤面积。

表 2 中对比了两种不同的加工损伤程度评价 方法, 分别是基于一维最大损伤长度的传统评价 方法以及基于加工损伤区域面积的新评价方法。 分析表明, 当加工损伤程度肉眼可辨时, 如 0.01 $\mathrm{mm} /$ tooth 和 $0.05 \mathrm{~mm} /$ tooth, $0.01 \mathrm{~mm} /$ tooth 和 0.10 $\mathrm{mm} /$ tooth 这两组情况, 损伤面积因子增量与每齿 进给增量的比值相对于一维损伤因子增量与每齿 进给增量的比值, 两者十分接近。但是, 对于损 伤程度相似的情况, 如每齿进给为 $0.05 \mathrm{~mm} /$ tooth 和 $0.10 \mathrm{~mm} / \mathrm{tooth}$ 这组情况, 损伤面积因子增量与 每齿进给增量的比值明显高于损伤长度增量与每 齿进给增量的比值。综上所述，基于损伤区域面 积, 是评价 CFRP 复合材料侧铣加工表层损伤程 度的一种行之有效的方法。

表 2 铁削试验参数及结果

\begin{tabular}{|c|c|c|c|c|c|c|c|c|}
\hline \multirow[b]{2}{*}{ No. } & \multicolumn{3}{|c|}{ 铣削参数 } & \multicolumn{3}{|c|}{ 损伤参数 } & \multicolumn{2}{|c|}{ 损伤因子 } \\
\hline & $\begin{array}{c}\text { 转速 } \\
n /(\mathrm{r} / \mathrm{min})\end{array}$ & $\begin{array}{c}\text { 每齿进给 } \\
f_{z} /(\mathrm{mm} / \text { tooth })\end{array}$ & $\begin{array}{c}\text { 径向切深 } \\
a_{\mathrm{e}} / \mathrm{mm}\end{array}$ & $\begin{array}{c}\text { 最大损伤深度 } \\
W_{\max } / \mathrm{mm}\end{array}$ & $\begin{array}{c}\text { 损伤区域面积 } \\
A_{\mathrm{D}} / \mathrm{mm}^{2}\end{array}$ & $\begin{array}{c}\text { 去除材料面积 } \\
A_{\mathrm{Q}} / \mathrm{mm}^{2}\end{array}$ & $\begin{array}{c}\text { 一维比长度 } \\
F_{d}\end{array}$ & $\begin{array}{c}\text { 二维比面积 } \\
F_{\mathrm{D}}\end{array}$ \\
\hline 1 & 2500 & 0.01 & 2.5 & 2.5 & 3.5834 & 125 & 1.00 & 0.0286672 \\
\hline 2 & 2500 & 0.05 & 2.5 & 4.0 & 65.7846 & 125 & 1.6 & 0.5262768 \\
\hline 3 & 2500 & 0.10 & 2.5 & 4.4 & 115.64538 & 125 & 1.76 & 0.92516304 \\
\hline 4 & 5000 & 0.01 & 2.5 & 2.5 & 3.36352 & 125 & 1.00 & 0.02690816 \\
\hline 5 & 5000 & 0.05 & 2.5 & 4.0 & 59.79448 & 125 & 1.60 & 0.47835584 \\
\hline 6 & 5000 & 0.10 & 2.5 & 4.4 & 101.71934 & 125 & 1.76 & 0.81375472 \\
\hline 7 & 10000 & 0.01 & 2.5 & 2.5 & 2.8 & 125 & 1.00 & 0.0224 \\
\hline 8 & 10000 & 0.05 & 2.5 & 4.0 & 51.24078 & 125 & 1.60 & 0.40992624 \\
\hline 9 & 10000 & 0.10 & 2.5 & 4.7 & 109.14466 & 125 & 1.88 & 0.87315728 \\
\hline
\end{tabular}

图 5 7 为 CFRP 复合材料侧铣加工后的图像, 以及提取出的损伤区域图像。其中, 图 5 为主轴转 速固定在 $2500 \mathrm{r} / \mathrm{min}$, 不同每齿进给下的加工损伤 情况。从图中可以清晰地看出, 随着每齿进给的增 加, 加工损伤区域明显增大。其中, 当每齿进给为 $0.01 \mathrm{~mm} /$ tooth 时, 加工后的 CFRP 复合材料表层未 见明显损伤、十分光滑; 但是, 当每齿进给增加到 $0.10 \mathrm{~mm} /$ tooth 时, 加工后的 CFRP 复合材料表层损 伤严重, 以形状不规则的毛刺为主, 部分已经扩展 到了被加工边缘的内侧, 形成了撕裂。其损伤面积 与每齿进给为 $0.01 \mathrm{~mm} /$ tooth 时的加工损伤程度相 比，损伤面积增长了近 32 倍。

如图 6 所示, 为主轴转速固定在 $5000 \mathrm{r} / \mathrm{min}$ 时
的 CFRP 复合材料表层加工损伤情况。从中可以看 出, 随着每齿进给的增大, 表层加工损伤程度亦显 著增加, 当采用小每齿进给 $(0.01 \mathrm{~mm} /$ tooth $)$ 铣削 CFRP 复合材料时, 被加工边缘依然光滑无毛刺; 相反, 当采用大每齿进给 $(0.10 \mathrm{~mm} /$ tooth $)$ 铣削时, CFRP 的表层损伤面积与采用小每齿进给铣削时相 比增长了近 30 倍, 这与转速为 $2500 \mathrm{r} / \mathrm{min}$ 时的情 况十分相似。

如图 7 所示, 为 $10000 \mathrm{r} / \mathrm{min}$ 下的 CFRP 复合 材料表层加工损伤情况。从图中可以看出, 10000 $\mathrm{r} / \mathrm{min}$ 时的表层加工损伤区域随每齿进给的变化规 律与主轴转速分别为 $2500 \mathrm{r} / \mathrm{min}$ 和 $5000 \mathrm{r} / \mathrm{min}$ 时的 加工损伤区域随每齿进给的变化规律基本一致, 即 

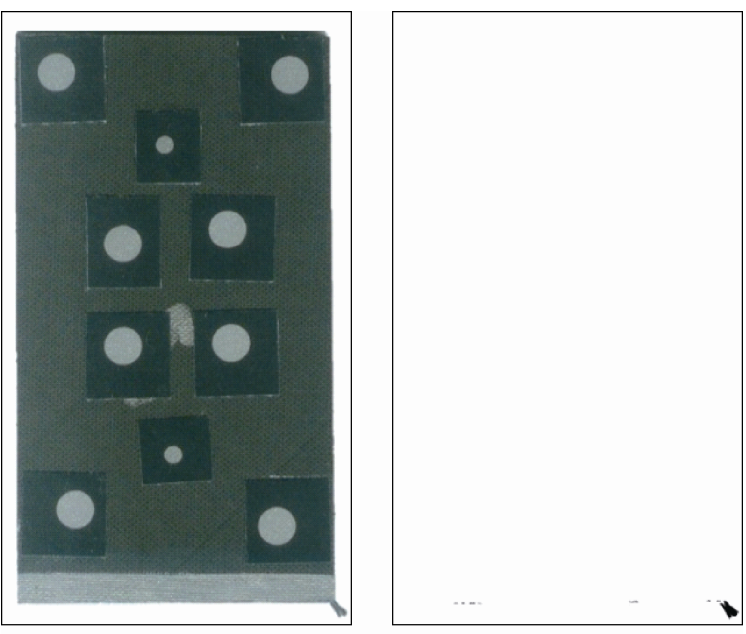

(a) $n=2500 \mathrm{r} / \mathrm{min}, f_{z}=0.01 \mathrm{~mm} /$ tooth
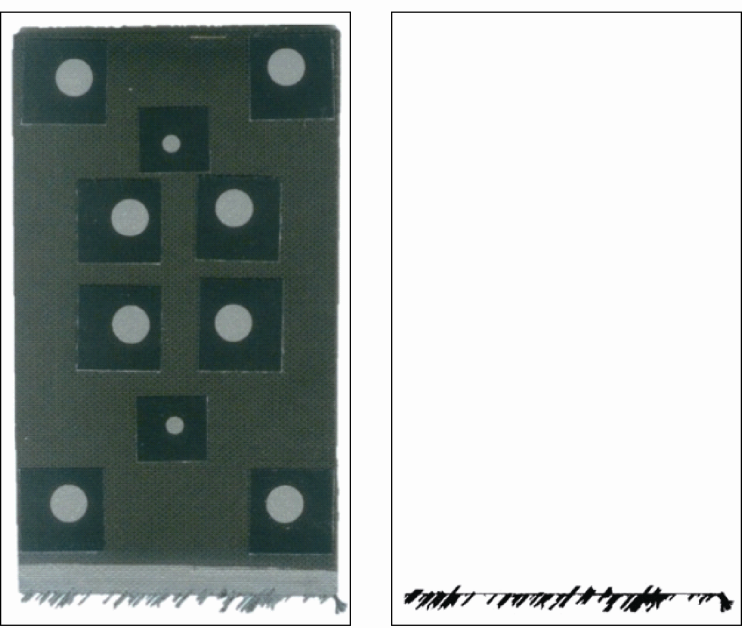

(b) $n=2500 \mathrm{r} / \mathrm{min}, f_{z}=0.05 \mathrm{~mm} / \mathrm{tooth}$
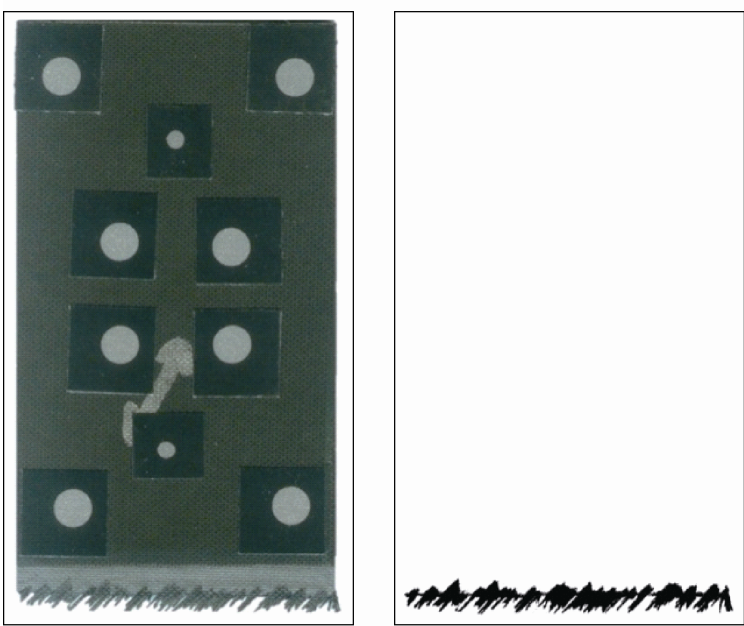

(c) $n=2500 \mathrm{r} / \mathrm{min}, f_{z}=0.10 \mathrm{~mm} /$ tooth

图 5 加工后的 CFRP 工件表面和提取出的损伤区域 (主轴转速 $2500 \mathrm{r} / \mathrm{min}$ )

无论转速如何变化, 加工损伤区域的面积均随着每 齿进给的增加而显著增大。由此可以推断出, 铣削 参数对表层加工损伤面积的影响程度由大到小依次 是每齿进给和主轴转速, 其中, 主轴转速对损伤面
积的影响并不明显。

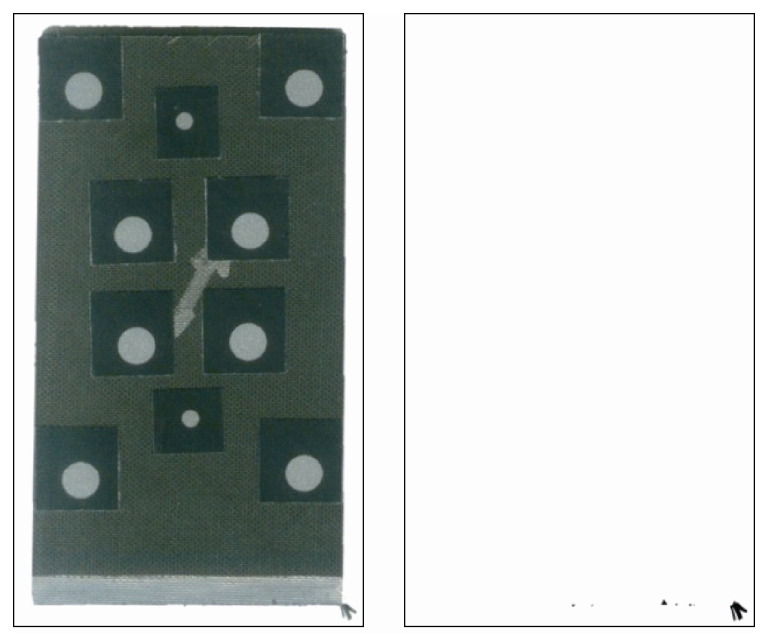

(a) $n=5000 \mathrm{r} / \mathrm{min}, f_{z}=0.01 \mathrm{~mm} / \mathrm{tooth}$
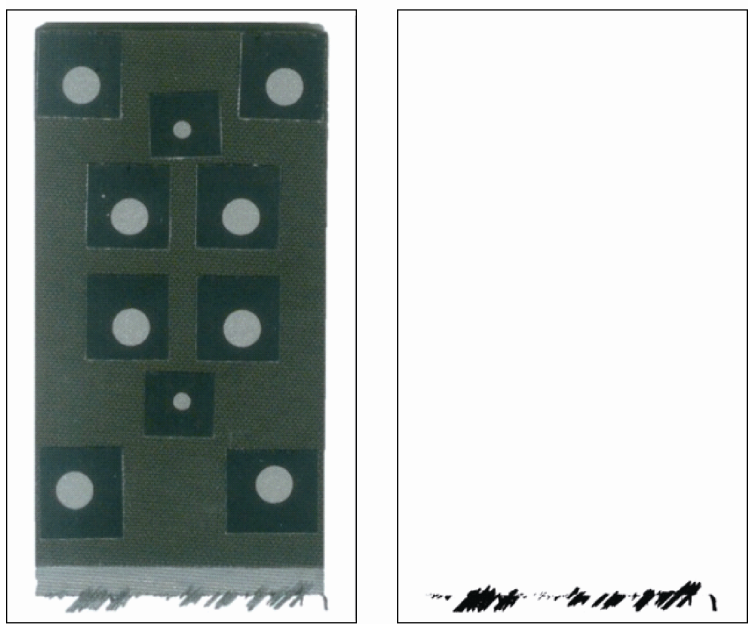

(b) $n=5000 \mathrm{r} / \mathrm{min}, f_{z}=0.05 \mathrm{~mm} / \mathrm{tooth}$
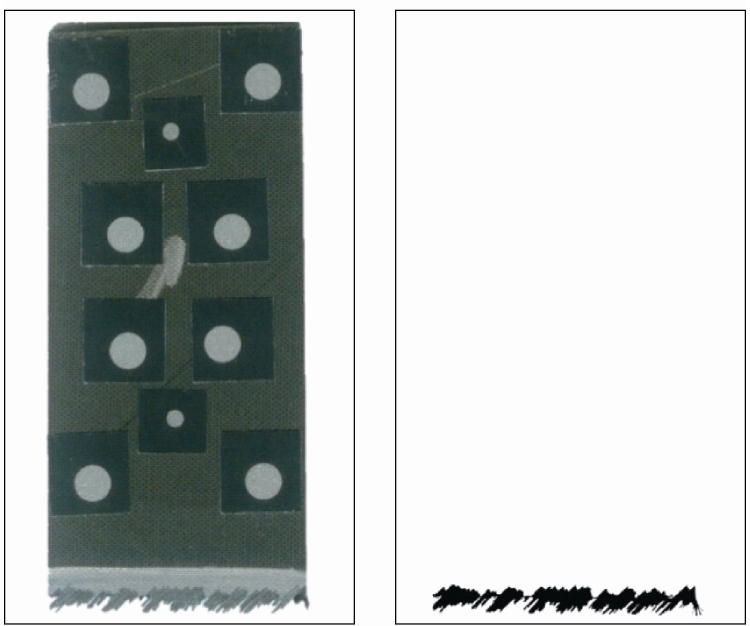

(c) $n=5000 \mathrm{r} / \mathrm{min}, f_{z}=0.10 \mathrm{~mm} /$ tooth

图 6 加工后的 CFRP 工件表面和提取出的损伤区域 (主轴转速 $5000 \mathrm{r} / \mathrm{min}$ )

\section{2 切削力变化规律}

切削力和切削热是影响 CFRP 复合材料表层加 工损伤程度的两个重要参数。切削力会导致切削热 的产生, 造成树脂基体软化, 减弱树脂对纤维的约 

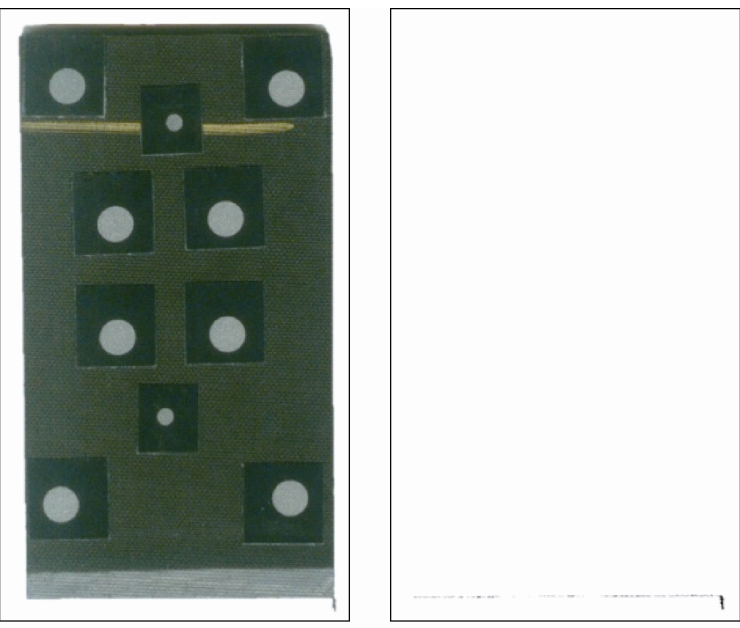

(a) $n=10000 \mathrm{r} / \mathrm{min}, f_{z}=0.01 \mathrm{~mm} /$ tooth

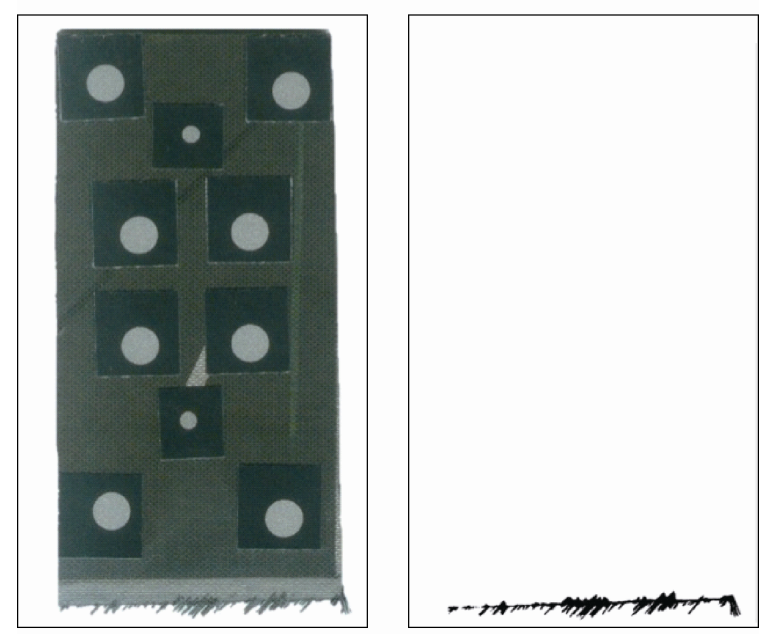

(b) $n=10000 \mathrm{r} / \mathrm{min}, f_{z}=0.05 \mathrm{~mm} /$ tooth
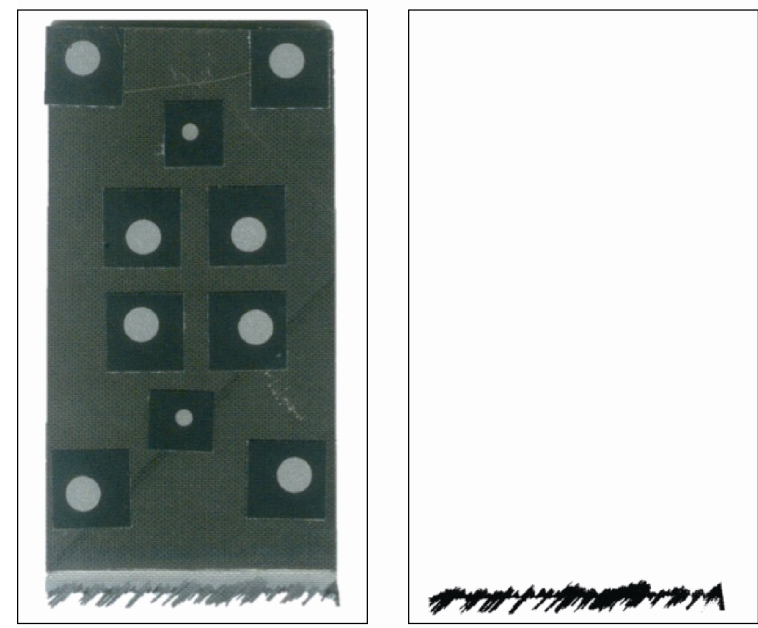

(c) $n=10000 \mathrm{r} / \mathrm{min}, f_{z}=0.10 \mathrm{~mm} /$ tooth

图 7 加工后的 CFRP 工件表面和提取出的损伤区域 (主轴转速 $10000 \mathrm{r} / \mathrm{min}$ )

束作用, 直接影响 CFRP 复合材料表层加工损伤 面积。因此, 研究切削力的变化规律, 对于揭示 表层损伤产生机制、降低加工引起的损伤具有重 要意义。
由于本文所使用的刀具为双刃直槽铣刀, 螺旋 角为 $0^{\circ}$ 。因此, 加工过程中不会产生沿刀具轴向 的铣削力分量, 如图 8 所示。刀具高速旋转过程中 容易引起机床主轴和夹具等的振动, 为了获得更为 准确的切削力数据, 采用低通滤波方法对原始切削 力信号进行处理, 以去除原始切削力信号中的杂 波, 效果如表 3、4 所示。从中可以看出, 滤波后 的切削力曲线与原始信号吻合较好, 幅值也没有出 现明显的衰减, 因此低通滤波对于抑制噪声的效果 十分明显。

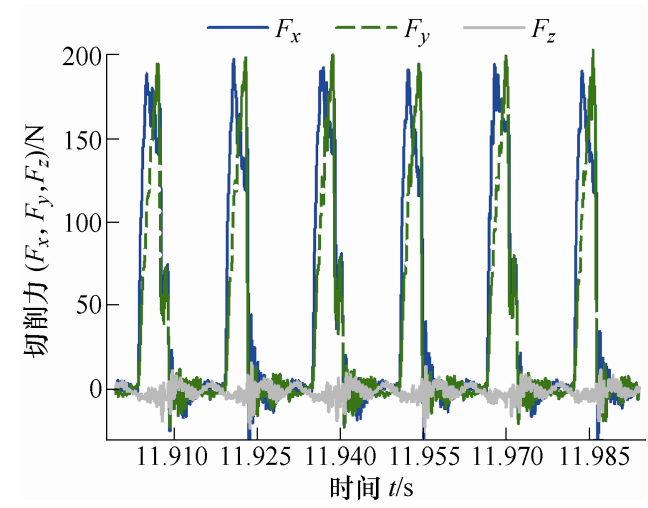

图 8 未经滤波处理的原始切削力数据

通过对侧铣加工过程中不同铣削参数下的最大 铣削力进行分析, 发现: 无论主轴转速如何变化, 最大 $y$ 轴切削力和最大 $x$ 轴切削力分量均表现出一 个随着每齿进给增加而升高的变化趋势。如图 9 所 示, $y$ 轴切削力和 $x$ 轴切削力随每齿进给的变化趋 势基本一致。当主轴转速为 $10000 \mathrm{r} / \mathrm{min}$ 时, 采用 $0.10 \mathrm{~mm} /$ tooth 进行铣削时的 $y$ 轴切削力相对于采用 $0.01 \mathrm{~mm} /$ tooth 时的 $y$ 轴切削力增长了近 $140 \%$ 。切 削力显然受到了每齿进给改变的影响, 进而在切削 力幅值上出现了显著的改变。然而, 随着主轴转速 增加, 铣削力的变化幅度明显小于每齿进给对铁削 力的影响程度, 说明主轴转速对铣削力的影响程度 比较小。因此, 铣削参数对铣削力的影响程度从大 到小依次是每齿进给和主轴转速。

\section{3 切削温度变化规律}

由于 CFRP 复合材料的导热性差, 其热导率仅 为 45 钢的 $1 / 16$, 加工过程中产生的热量不易向周围 介质传出, 所引起的高温又易超过树脂的玻璃转化 温度, 导致树脂约束纤维的能力下降, 从而加剧损 伤的产生 ${ }^{[23]}$ 。因此, 在时域内同步测量得到切削温 度的变化规律, 有助于深入地了解和研究 CFRP 复 合材料表层加工损伤产生机制。 
表 3 滤波后的主切削力信号波形

\begin{tabular}{|c|c|c|c|}
\hline \multirow{2}{*}{ 进给速度 } & \multicolumn{3}{|c|}{ 转速 } \\
\hline & $2500 \mathrm{r} / \mathrm{min}$ & $5000 \mathrm{r} / \mathrm{min}$ & $\min$ \\
\hline $\mathrm{mm} /$ tooth & 滤波前 & 滤波前 & 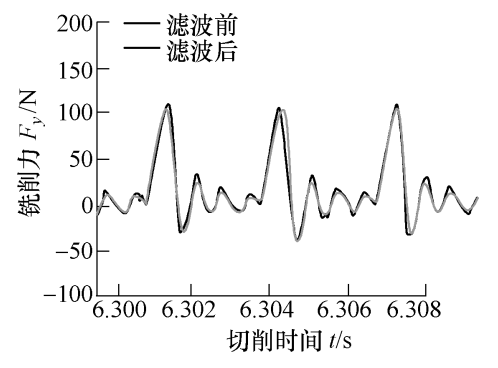 \\
\hline $\mathrm{mm} /$ tooth & 滤波前 & 滤波前 & 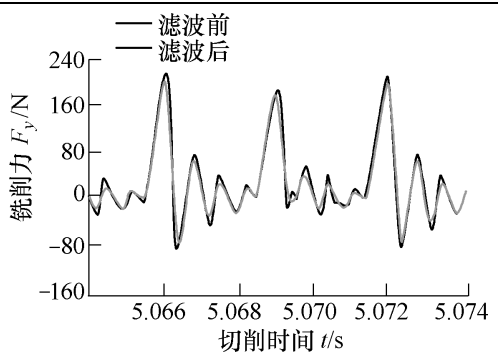 \\
\hline $\mathrm{mm} /$ tooth & 滤波前 & 二滤波前 & 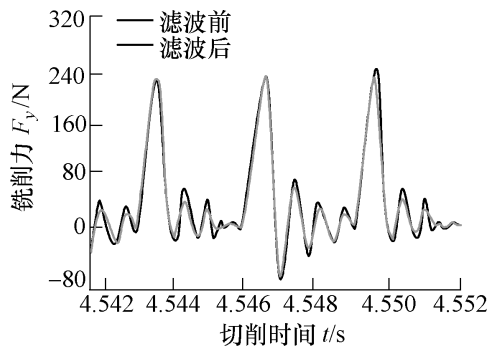 \\
\hline
\end{tabular}

\section{表 4 滤波后的径向切削力信号波形}

\begin{tabular}{|c|c|c|c|}
\hline \multirow{2}{*}{ 进给速度 } & \multicolumn{3}{|c|}{ 转速 } \\
\hline & $2500 \mathrm{r} / \mathrm{min}$ & $5000 \mathrm{r} / \mathrm{min}$ & $10000 \mathrm{r} / \mathrm{min}$ \\
\hline $\mathrm{mm} /$ tooth & 海波前 & 滤波前 & 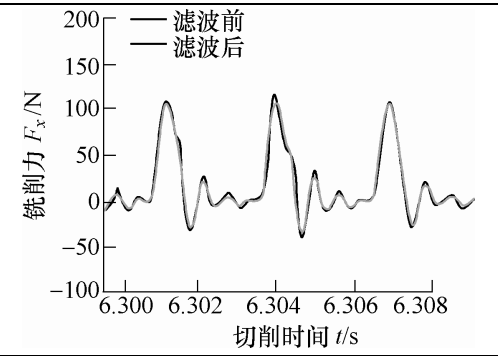 \\
\hline 0.05 & 滤波前 & 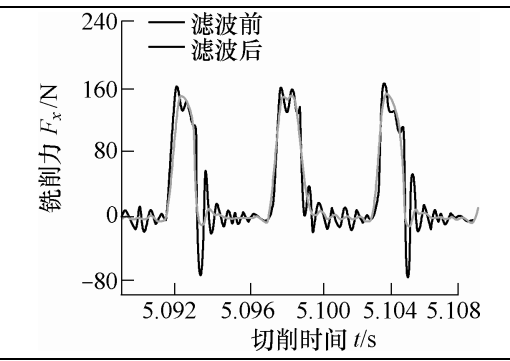 & 5.066 切 \\
\hline 0.10 & 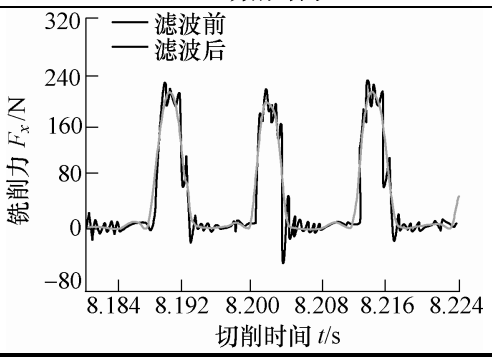 & 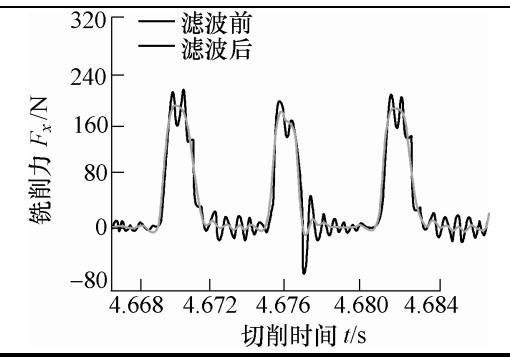 & $\begin{array}{ll}320 \\
240 \\
2\end{array}$ \\
\hline
\end{tabular}




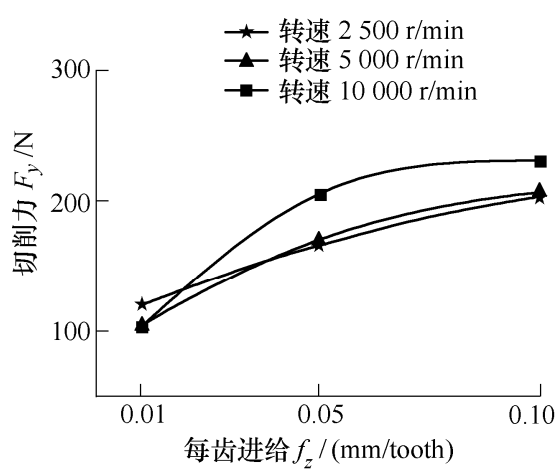

(a) $y$ 轴切削力

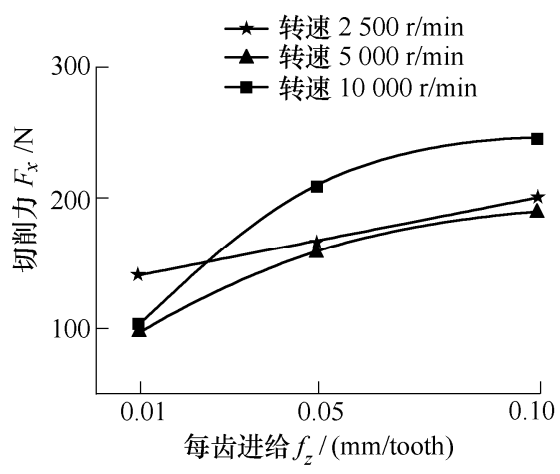

(b) $x$ 轴切削力

图 9 切削力各分量随每齿进给的变化规律曲线

CFRP 复合材料铣削过程中, 由热成像仪实时 采集记录的切削温度数据如图 10 所示。从图中可以 看出, 最高切削温度位于刀具即将切出时的位置处。 这一现象与热累积效应有关, 即在下一次切削开始 之前, 上一次切削引起的温度无法在短时间内冷却 到室温, 加之铣削过程的特殊性, 使得温度不断累 积, 导致切削温度时变曲线持续㣏升。通过对不同 铣削参数下的切削温度时变曲线进行分析, 发现: 当每齿进给从 $0.01 \mathrm{~mm} / \mathrm{tooth}$ 增加到 $0.10 \mathrm{~mm} / \mathrm{tooth}$ 时, 无论转速如何变化, 最高温度均表现出一个随 着每齿进给增加而降低的变化趋势。

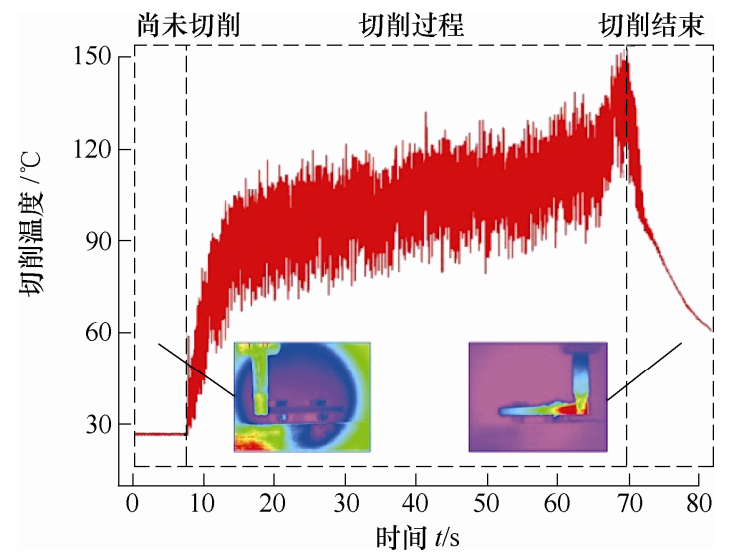

图 10 切削温度时变曲线

从图 11 所示可以清晰地看出, 主轴转速为
$2500 \mathrm{r} / \mathrm{min}$ 时的最高温度值均低于主轴转速为 $5000 \mathrm{r} / \mathrm{min}$ 和 $10000 \mathrm{r} / \mathrm{min}$ 时的最高温度值。产生 这一现象的主要原因是, 当每齿进给保持不变时, 单位时间内刀具和工件之间的摩擦长度会随着主轴 转速的增大而增加 ${ }^{[24]}$, 使得加工过程中的总产热量 增加, 切削温度相应地也会升高。当主轴转速固定 不变时, 采用小每齿进给也会使得切削温度升高, 这主要是由于在相同转速条件下, 减小每齿进给会 增加刀具和工件之间的相互摩擦时间, 切削过程的 总产热量也会随之增加。

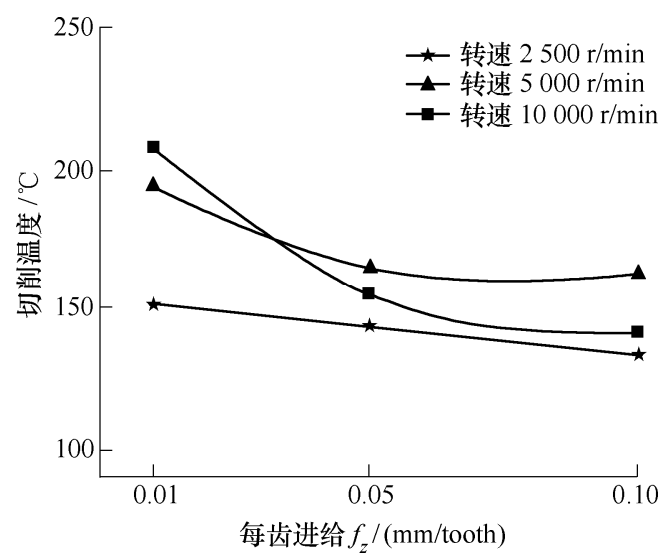

图 11 最高切削温度随每齿进给的变化规律曲线

\section{4 力热数据与表层损伤面积的相关性分析}

通过进一步分析，发现采用小每齿进给时的温 升速率明显小于采用大每齿进给时的温升速率。以 转速 $2500 \mathrm{r} / \mathrm{min}$ 为例, 从室温升高到 $70^{\circ} \mathrm{C}$, $0.01 \mathrm{~mm} /$ tooth 所需的时间约为 $3.742 \mathrm{~s}$, 而采用 $0.10 \mathrm{~mm} /$ tooth 仅需 $0.58 \mathrm{~s}$ 。温升的速率越快, 意味 着热冲击越大, 随之发生的热软化率也相应增加, 形成的局部高温会导致树脂在短时间内被均匀软 化。因此, 采用小每齿进给, 可以降低热软化率、 减少切削热对加工过程的影响。

如图 12 所示, 通过对最大切削力和温升速率的 数据进行分析不难发现, 在加工过程中切削力与温 升速率的变化趋势基本保持一致, 且切削力越大,

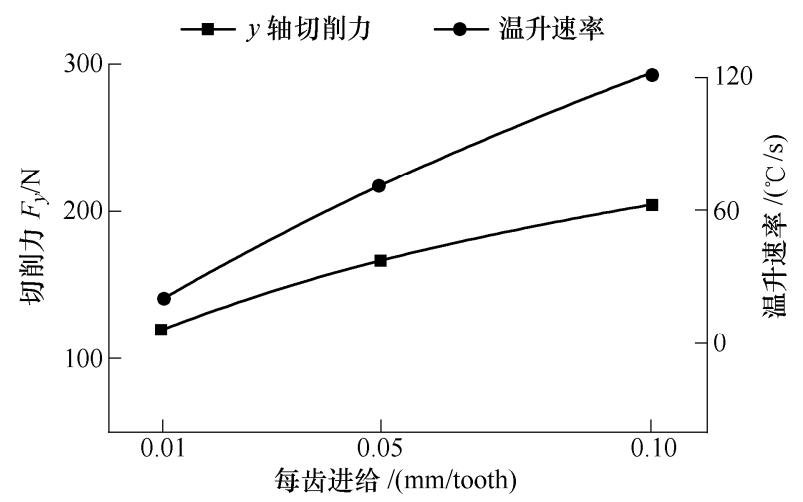

图 12 最大切削力和温升速率对比分析 
对应的温升速率亦越快。而在相同切削条件下, 随 着每齿进给的增加, 相似的变化趋势在最大切削力 和表层损伤面积因子之间被观察到, 如图 13 所示, 为 $5000 \mathrm{r} / \mathrm{min}$ 铣削 CFRP 复合材料时的表层加工损 伤面积和 $y$ 轴切削力对比分析结果。这一发现同样 适用于转速为 $2500 \mathrm{r} / \mathrm{min}$ 和 $10000 \mathrm{r} / \mathrm{min}$ 时的情况。

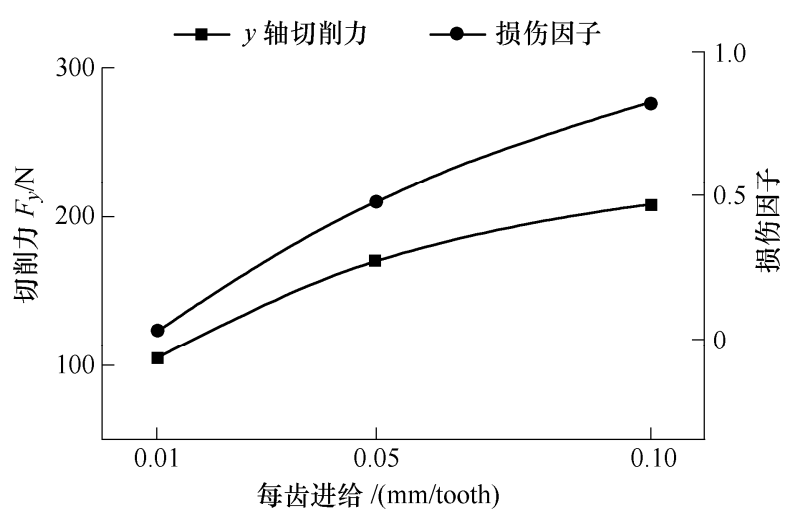

图 13 最大切削力和表层加工损伤面积因子对比分析

综上所述, 温升速率、最大切削力和表层损伤 面积因子三者均随着每齿进给的增大而增加, 说明 表层损伤面积因子与最大切削力、温升速率之间存 在着明显的内在联系。由此可以得出一个显然的结 论, 最大切削力越大, 对应的温升速率越快, 最终 导致加工表层损伤面积因子也越大。这主要是由于 表层材料易在切削力和切削温度共同作用下发生垂 直于板厚方向的挠曲变形, 在变形过程中易形成III 型裂纹并发生扩展 ${ }^{[25]}$, 最终导致 CFRP 复合材料形 成毛刺、撕裂等表层损伤。由此可以推断出, 通过 降低温升速率或者降低最大切削力, 均可以达到减 小加工表层损伤面积因子的目的。因此, 本文推荐 使用小每齿进给即通过减少单次切削厚度的方式加 工 CFRP 复合材料。如果为了保证加工效率, 需要 通过冷却工艺降低温升速率, 控制切削温度在 CFRP 复合材料的最适温度区间内。

\section{4 结论}

本研究主要贡献为基于数字图像处理技术, 建 立了 CFRP 复合材料铣削加工表层损伤面积评价方 法, 阐明了铣削参数与表层损伤面积、切削力、切 削温度之间的影响关系, 获得了力热共同作用下的 CFRP 表层损伤成因及其抑制方法, 主要结论如下。

（1）基于加工损伤区域面积的评价方法明显优 于传统一维损伤评价方法, 可以有效避免传统方法 因只能考虑损伤的一维长度信息无法评价加工损伤 程度大小的问题。

(2) CFRP 复合材料的表层加工损伤面积受每齿
进给变化的影响显著, 主轴转速的影响次之。采用 小每齿进给铣削时, 无论主轴转速如何变化, 表层 均未见明显损伤、十分光滑。

（3）最大切削力、温升速率和表层损伤面积因 子均随着每齿进给的增大而增加, 最大切削力越大, 对应的温升速率也越快, 最终导致加工表层损伤面 积因子也越大。

(4) 实际加工中, 推荐使用小每齿进给对 CFRP 复合材料进行加工, 如果为了保证加工效率, 通过 冷却工艺降低温升速率、控制切削温度在合适区间 是抑制加工表层损伤的一种有效途径。

\section{参 考 文 献}

[1] 杜善义. 先进复合材料与航空航天 [J]. 复合材料学报, 2007(1): 1-12.

DU Shanyi. Advanced composite materials and aerospace engineering[J]. Acta Materiae Compositae Sinica, 2007(1): 1-12.

[2] CHE Demeng, SAXENA I, HAN Peidong, et al. Machining of carbon fiber reinforced plastics/polymers: a literature review[J]. Journal of Manufacturing Science \& Engineering, 2014, 136(3): 034001.

[3] LIU Defu, TANG Yongjun, CONG Weilong. A review of mechanical drilling for composite laminates[J]. Composite Structures, 2012, 94(4): 1265-1279.

[4] STONE R, KRISHNAMURTHY K. A neural network thrust force controller to minimize delamination during drilling of graphite-epoxy laminates[J]. International Journal of Machine Tools \& Manufacture, 1995, 36(9): 985-1003.

[5] HOCHENG H, DHARAN C K H. Delamination during drilling in composite laminates[J]. Transactions-American Society of Mechanical Engineers Journal of Engineering for Industry, 1990, 112(3): 236-239.

[6] TSAO C C, HOCHENG H. Effect of eccentricity of twist drill and candle stick drill on delamination in drilling composite materials[J]. International Journal of Machine Tools \& Manufacture, 2005, 45(2): 125-130.

[7] JAIN S, YANG D C H. Effects of feedrate and chisel edge on delamination in composites drilling[J]. Transactions-American Society of Mechanical Engineers Journal of Engineering for Industry, 1993, 115: 398-398.

[8] GURURAJA S, RAMULU M. Modified exit-ply delamination model for drilling FRPs[J]. Journal of Composite Materials, 2009, 43(5): 483-500.

[9] HINTZE W, HARTMANN D. Modeling of delamination during milling of unidirectional CFRP[J]. Procedia CIRP, 2013, 8: 444-449. 
[10] 周井文, 陈燕, 傅玉灿, 等. 纤维切削角对 CFRP 加工 缺陷的影响规律 [J]. 哈尔滨工业大学学报, 2015, 47(7): 110-116.

ZHOU Jingwen, CHEN Yan, FU Yucan, et al. Influence of fiber cutting angle on the machining defects during slotting of CFRP[J]. Journal of Harbin Institute of Technology, 2015, 47(7): 110-116.

[11] SOLDANI X, SANTIUSTE C, MUÑOZ-SÁNCHEZ A, et al. Influence of tool geometry and numerical parameters when modeling orthogonal cutting of LFRP composites[J]. Composites Part A: Applied Science and Manufacturing, 2011, 42(9): 1205-1216.

[12] BHATNAGAR N, NAYAK D, SINGH I, et al. Determination of machining-induced damage characteristics of fiber reinforced plastic composite laminates[J]. Materials and Manufacturing Processes, 2004, 19(6): 1009-1023.

[13] RAO G $\mathrm{V}$ G, MAHAJAN P, BHATNAGAR $\mathrm{N}$. Micro-mechanical modeling of machining of FRP composites-Cutting force analysis[J]. Composites Science and Technology, 2007, 67(3): 579-593.

[14] CALZADA K A, KAPOOR S G, DEVOR R E, et al. Modeling and interpretation of fiber orientation-based failure mechanisms in machining of carbon fiber-reinforced polymer composites[J]. Journal of Manufacturing Processes, 2012, 14(2): 141-149.

[15] 殷俊伟, 贾振元, 王福吉, 等. 基于 CFRP 切削过程仿 真的面下损伤形成分析 [J]. 机械工程学报, 2016, 52(17): 58-64.

YIN Junwei, JIA Zhenyuan, WANG Fuji, et al. FEM Simulation Analysis of Subsurface Damage Formation Based on Continuously Cutting Process of CFRP[J]. Journal of Mechanical Engineering, 2016, 52(17): 58-64.

[16] YASHIRO T, OGAWA T, SASAHARA H. Temperature measurement of cutting tool and machined surface layer in milling of CFRP[J]. International Journal of Machine Tools and Manufacture, 2013, 70: 63-69.

[17] BRINKSMEIER E, FANGMANN S, RENTSCH R. Drilling of composites and resulting surface integrity[J]. CIRP Annals-Manufacturing Technology, 2011, 60(1): 57-60.

[18] KIM H S, WANG Wenxue, TAKAO Y. Evaluation by FEM of temperature-dependent damage behavior in quasi-isotropic carbon/epoxy laminates[J]. Advanced Composite Materials, 1999, 8(3): 247-257.

[19] PECAT O, RENTSCH R, BRINKSMEIER E. Influence of milling process parameters on the surface integrity of CFRP[J]. Procedia CIRP, 2012(1): 466-470.

[20] 张厚江, 陈五一, 陈鼎昌. 碳纤维复合材料(CFRP)钻孔出 口缺陷的研究[J]. 机械工程学报, 2004, 40(7): 150-155.

ZHANG Houjiang, CHEN Wuyi, CHEN Dingchang. Investigation of the exit defects in drilling carbon fiber-reinforced plastic plates[J]. Journal of Mechanical Engineering, 2004, 40(7): 150-155.

[21] 周正干, 孙广开, 李征, 等. 复合材料层压板钻孔分层 激光超声检测方法[J]. 机械工程学报，2013，49(22): 29-33.

ZHOU Zhenggan, SUN Guangkai, LI Zheng, et al. Laser ultrasonic detection of drilling-induced delamination in composite laminates[J]. Journal of Mechanical Engineering, 2013, 49(22): 29-33.

[22] DAVIM J P, REIS P. Damage and dimensional precision on milling carbon fiber-reinforced plastics using design experiments[J]. Journal of Materials Processing Technology, 2005, 160(2): 160-167.

[23] 王奔, 高航, 郭东明. 树脂固化温度与纤维铺设方式对 C/E 复合材料制孔质量的影响 $[\mathrm{J}]$. 机械工程学报, 2011, 47(12): 19-25.

WANG Ben, GAO Hang, GUO Dongming. Influence of resin cure temperature and fiber lay-up style on the drilling quality of $\mathrm{C} / \mathrm{E}$ composites[J]. Journal of Mechanical Engineering, 2011, 47(12): 19-25.

[24] CONG Weilong, ZOU Xiaotian, DEINES T W, et al. Rotary ultrasonic machining of carbon fiber reinforced plastic composites: An experimental study on cutting temperature[J]. Journal of Reinforced Plastics and Composites, 2012, 31(22): 1516-1525.

[25] WANG Fuji, YIN Junwei, MA Jianwei, et al. Effects of cutting edge radius and fiber cutting angle on the cutting-induced surface damage in machining of unidirectional CFRP composite laminates[J]. The International Journal of Advanced Manufacturing Technology. 2017, 91(9): 3107-3120.

作者简介: 王福吉, 男, 1974 年出生, 博士，教授，博士研究生导师。 主要研究方向为复合材料去除机理与损伤成因。

E-mail: wfjsll@dlut.edu.cn

殷俊伟, 男, 1986 年出生, 博士研究生。主要研究方向为复合材料加工 损伤形成机制及其抑制技术。

E-mail: dlyjw@mail.dlut.edu.cn

贾振元, 男, 1963 年出生, 博士, 教授, 博士研究生导师, 长江学者特 聘教授。主要研究方向为复合材料构件加工理论与工艺。

E-mail: jzyxy@dlut.edu.cn

马建伟(通信作者), 男, 1984 年出生, 博士, 副教授。主要研究方向为 难加工材料高速铣削。

E-mail: mjw2011@dlut.edu.cn 